Results The scenario scores increased from an average of $81.75 \%$ to $96.75 \%$. The audit scores increased from $63 \%$ to $100 \%$.

Conclusions A combination of the comprehensive guidance, including the Post Falls Pathway and staff education (including how to safely manoeuvre a patient after a fall), has demonstrated an increase in confidence and consistency of assessment and has reduced the risk of harm. Our approach has demonstrated that the introduction of the pathway has significantly improved the safe management of patients who have experienced a fall.

\section{P-146 OUR QUALITY IMPROVEMENT JOURNEY AT HOSPICE ISLE OF MAN: SMALL CHANGES NEED SMALL STEPS}

Tracy Broom, David Waters, Cheryl Young, Ben Harris, Anne Mills. Hospice Isle of Man, Douglas, Isle of Man

\subsection{6/spcare-2021-Hospice.163}

Background In June 2021, the hospice began delivering our first ever Quality Improvement (QI) Programme. We had observed an environment that was innovative in research and could be innovative in QI. We all 'improved quality' but did we use QI as a framework for that improvement?

Aims Our shared vision was to integrate QI into the normal working day, within a culture of continuous improvement (National Advisory Group on the Safety of Patients in England, 2013). We have now launched our QI journey with our first sequential and small steps; a QI Programme and QI Champions.

Methods The programme was delivered over four days to clinical and non-clinical staff; motivated and energetic people from every department in Hospice. We delivered QI technical skills (Institute for Healthcare Improvement. How to improve [The Model for Improvement]) blending theory and practice, whilst also focusing on wider topics which support QI landing successfully in the day job. This included improvement leadership (Øvretveit, 2009), human factors (National Quality Board, 2013) and team work (Montgomery, Parkin, Chisholm, et al., 2020). These specific topics were chosen by the participants, so the programme was bespoke to their learning needs and overall the programme was designed to be a fun and interactive environment.

Results We commenced evaluation with a pre-course selfassessment of QI knowledge, skills and confidence. This was repeated at the end of each facilitated day and will be concluded by a post-course self-assessment. The first cohort completes in September 2021 and we will have the programme evaluation completed the same month.

Conclusions We anticipate participants will have improved QI knowledge, skills and confidence which will enable them to actively use QI in their normal working day and will also transition to become QI Champions. The QI Champion is a new role being designed to support other staff, encourage momentum and be a key part of building a network of QI Champions. We would like to share our story so far and future system plans. Sharing, learning, improving.

\section{P-147 ABSTRACT WITHDRAWN}

\section{P-148 INPATIENTS HOSPICE ADMISSIONS, WHO IS ADMITTED AND WHY: A MIXED METHOD PROSPECTIVE STUDY}

1,2Erna Haraldsdottir, ${ }^{3}$ Libby Milton, ${ }^{1}$ Anna Lloyd, ${ }^{3,4}$ Anne Finucane, ${ }^{3}$ Martyn Bijak, ${ }^{1} J a c k i e$ Stone, ${ }^{1}$ Dot Partington, ${ }^{3}$ Hilary Ford, 'Duncan Brown. 'St Columba's Hospice Care, Edinburgh, UK; ${ }^{2}$ Queen Margaret University, Edinburgh, UK; ${ }^{3}$ Marie Curie Hospice, Edinburgh, UK; ${ }^{4}$ University of Edinburgh, Edinburgh, UK

\subsection{6/spcare-2021-Hospice. 164}

Background Across the UK, more people are projected to die in community settings over the next two decades. The role of the hospice inpatient unit (IPU) needs to be better understood in light of these shifts. The term complex needs is used for patients admitted to IPU, however, there is little clarity around these needs and how they trigger admission.

Aim To understand why patients are admitted to IPU and to describe the palliative care services available to them beforehand.

Method Prospective mixed methods study. Data were collected in two hospices in one city of Scotland. We examined case notes of 259 patients admitted to the IPU over four months and conducted 40 semi-structured interviews. (22 patients or a relative proxy, 11 health care professionals, 7 pro-forma interviews-source of referrals).

Results Mean age was 71 years, 53\% were female; 47\% male. Most patients admitted were Scottish or White British (95\%). Most were living with another person at the time of admission (72\%); 28\% lived alone. The vast majority had cancer (95\%). Phase of illness was judged as deteriorating or unstable for over two-thirds at the time of admission. Most patients were receiving specialist palliative care support prior to admission $-73 \%$ had a community palliative care CNS (Clinical Nurse Specialist). Just under half had district nurse support (48\%). Over one-fifth (21\%) had no prior community palliative care involvement; most of these patients were referred from the hospital (81\%). Length of stay was 12 days (median). 68\% of admissions were for end-of-life care, with the patient dying during the admission. The hospice inpatient unit was the documented preferred place of death for $56 \%$ who died in that location.

Thematic analysis of the qualitative data identified the key reasons for admissions, symptom control, anxiety and fear, social isolation and end-of-life care.

\section{Conclusion}

- Admission to inpatient hospice is a last resort and often a response to a crisis situation.

- Greater palliative care support in home settings is needed so that more patients can remain at home when that is their preference.

\section{P-149 A REVIEW OF OUTCOME MEASURES USED BY SPEECH AND LANGUAGE THERAPISTS IN ADULTS UNDER PALLIATIVE CARE}

${ }^{1}$ Charlotte Robinson, ${ }^{2}$ Eli Harriss, ${ }^{1}$ Mary Miller. ' Oxford University Hospitals NHS Foundation Trust, Oxford, UK; ${ }^{2}$ Bodleian Library, Oxford University, Oxford, UK

\subsection{6/spcare-2021-Hospice. 165}

Introduction Little is known about the use of outcome measures $(\mathrm{OM})$ by Speech and Language Therapists (SLTs) in palliative care. This review aimed to identify studies using validated $\mathrm{OMs}$ in adults receiving palliative care, and to 
understand whether the OMs captured the impact of SLT interventions.

Methods The Preferred Reporting Items for Systematic Reviews and Meta Analyses (PRISMA) guidelines were used. 422 items were screened, 308 met the inclusion and exclusion criteria and seven papers were selected for review.

Results The papers described 296 patients. (range of 1 - 96 participants per study), aged 19 - 89 years, diagnosed with cancer (83), motor neurone disease (4), other/mixed (208), progressive neurological (1) and not further described (2). The heterogeneity of the study designs did not allow for further analysis. The studies were of poor quality.

Ten outcome measures were used in the studies. Assessment at two time points were described in 14 patients (two studies).

Conclusion OMs commonly used in palliative care do not address the main areas of clinical focus of SLTs; dysphagia and communication barriers. OMs used in SLT practice are rarely validated in palliative populations. Challenges are compounded by the reality of practice in the UK where SLTs offer consultation for palliative care patients but are infrequently employed within palliative care teams. Contact with patients may be a single assessment and advice session, reducing the opportunity to use OMs and demonstrate the efficacy of SLT colleagues. Evidence of change in response to SLT intervention is not yet demonstrated in the literature. Use of validated OMs by SLTs in palliative care appears to lag behind use of OMs in other areas of SLT work, and behind use of OMs by other professionals in palliative care. Development and use of validated OMs should consider flexibility as patient function changes and the need for both patient and proxy completion.

\section{P-150 I ALWAYS ASSESS MY PATIENTS HOLISTICALLY I DON'T NEED A SCORING SYSTEM TO TELL ME HOW ILL THEY ARE'}

Cheryl Young, Lonan A Challis, Sarah M McGhee, Anne Mills. Hospice Isle of Man, Douglas, Isle of Man

\subsection{6/spcare-2021-Hospice. 166}

Background Measuring patient outcomes is necessary to assess the impact of care and identify areas for improvement (Etkind, Daveson, Kwok, et al., 2015; Marshall, Haywood, Fitzpatrick, 2006). It is therefore vital that staff are confident and comfortable using outcome measures. Hospice adopted three of the Outcome Assessment and Complexity Collaborative (OACC) (Witt, Murtagh, de Wolf-Linder, et al.) measures in July 2019: the Phase of Illness, Australia-modified Karnofsky Performance Status (AKPS) and Integrated Palliative care Outcome Scale (IPOS). In July 2020, Hospice investigated clinicians' perceptions of the measures.

Aim To understand clinicians use and views of the OACC measures, any problems and suggestions for improvement.

Methods A survey was distributed to clinicians $(n=42)$. Descriptive statistics were calculated using the statistical packages R and RStudio (Version 4.1.0 for Windows). Qualitative data were analysed using a thematic analysis framework (Braun \& Clarke, 2006).

Results Twenty-nine clinicians (response rate 69\%) had used one of the three measures at least once. Twenty-five (93\%), $22(85 \%)$ and $24(92 \%)$ stated that they use IPOS, AKPS and Phase of Illness some or all of the time, respectively.
Moreover, 24 (83\%), 23 (79\%) and 21 (72\%) clinicians felt confident appropriately using IPOS, AKPS and Phase of Illness, respectively.

Respondents identified the measures as a helpful resource for person-centred assessment and monitoring. They can help open up dialogue and increase rapport between patient and clinician, and provide a 'common language' between clinicians. However, they felt the measures added little to a clinical assessment, particularly for Allied Health Professionals (AHPs), and an apparent focus on physical health limited holistic assessment.

Respondents recommended increased application of OACC measures at handover. For AHPs, other outcome measures might be considered. Some wanted feedback on outcomes and more training on use of the measures.

Conclusions In a palliative care setting, benefits were witnessed mainly in patient assessment but less in the application of outcomes. Further staff training and application of outcomes may be beneficial.

\section{P-151 LEARNING FROM DEATHS IN HOSPICE CARE}

Nick Dando, Jayne Holland, Cate Seton-Jones. Phyllis Tuckwell Hospice Care, Farnham, UK

\subsection{6/spcare-2021-Hospice. 167}

Background The NHS National Quality Board published its first edition of 'National Guidance on Learning from Deaths' in March 2017. Although a charitable hospice care provider, Phyllis Tuckwell Hospice Care (PTHC) followed NHS, Care Quality Commission (2016, 2018) and Royal College of Physicians (2016) guidance and established a pilot process for learning from deaths in 2018.

Aims This report outlines the approach taken to establish a new process and culture of learning from deaths in a UK hospice and shares our experience as a model of good practice.

Methods Terms of Reference (TOR) were established to guide the structure and the culture of the learning from deaths process. The TOR linked learning from deaths with PTHC's core values including keeping patient care at the heart of our practice, acting with honesty and integrity and fostering a culture of continuous learning.

Results The first Learning from Deaths review occurred in December 2018 following the death of a patient during transfer to hospital for acute treatment. An outcome of this meeting was to review the care of three further patients who were transferred to the acute sector. Learning translated directly into a change in clinical practice in August 2019 through the roll-out of the National Early Warning Score 2 (Royal College of Physicians, 2017; Frinton, Malia, Owen, et al., 2019) on the inpatient unit. The learning from deaths approach is now integrated into PTHC's clinical governance system and 19 cases have been reviewed between April 2019 and March 2021.

Conclusions Conducting formal reviews into the deaths of patients under hospice care offers an important opportunity for reflection and learning to improve services for future patients. Senior support and a constructive, positive culture are essential facilitators in establishing staff engagement with the process. A literature review did not identify any reports of existing models for learning from deaths in hospice care. This innovative approach is transferrable to other charitable units. 